\title{
STUDY OF MASS-LOSS IN THE PLANETARY NEBULA NGC 1514
}

\author{
B. Aryal*, A. Mishra* and R. Weinberger*** \\ *Central Department of Physics, Tribhuvan University, Kathnamdu, Nepal. \\ ***Institut für Astrophysik, Leopold-Franzens-Universität Innsbruck, Austria.
}

\begin{abstract}
The planetary nebula NGC 1514 is found to reside within giant dust structures which may represent fossil records of its progenitor's transition from spherically symmetric to bipolar mass loss. The transition from spherically symmetric Asymptotic Giant Branch (AGB) mass loss to aspherical Planetary Nebulae (PNe) is an intriguing problem of stellar astrophysics. On $12 \mu \mathrm{m}$ maps of the Infrared Astronomical Satellite (IRAS) we detected a huge (2.6 pc) roundish emission nebula around the evolved PNNGC 1514. On 100 and $60 \mu \mathrm{m}$ IRAS maps we additionally found two giant (12 pc) bipolar dust emission structures centered on NGC 1514. The total mass of all these structures is $2.2 \pm 1.4$ solar masses. We argue that NGC 1514 and its dusty surroundings represent the preserved history of the main mass loss phases of a star of intermediate initial mass.
\end{abstract}

Keywords: Interstellar medium; Infrared astronomy; Planetary nebula; Stars; Asymptotic giant branch.

\section{INTRODUCTION}

Towards the finale of their lifetime, almost all (95-98\%) stars, that is stars of low and intermediate initial mass $\left(M_{\omega} \sim 1-8\right.$ solar mass) lose a substantial fraction of their mass on the Asymptotic Giant Branch (AGB) in form of massive winds, which compels them into the Planetary Nebula (PN) phase. PNe are well known for their fascinating range of shapes and morphologies. Besides being ideal laboratories for the study of various astrophysical processes prevailing in highly excited dilute nebulae, $\mathrm{PNe}$ and their progenitors are key objects for the understanding of the evolution of stars $(2,2)$. In this connexion, the transformation from spherically symmetric $\mathrm{AGB}$ winds to non-spherical PNe represents one of the most enduring problems of stellar astrophysics (3). Obviously, high-speed collimated outflows of jets acting during the late $\mathrm{AGB}$ or proto-planetary nebula (PPN) phase are responsible for the non-sphericity and thus play a decisive role in shaping PNe (4-6). Multitudinous observations of AGB stars, PPNe and $\mathrm{PNe}$ have been serving as snapshots in time in order to understand the origin and evolution of an average (aspherical) PN. Despite all these great efforts the transition from $A G B$ stars - the main producers of carbonaceous stardust (7) - to PNe however is still poorly understood $(8,9)$.

\section{OBSERVATION}

Here we report on giant dust structures found in the far infrared around the evolved planetary nebula NGC 1514, on maps of the Infrared Astronomical Satellite (IRAS). NGC 1514 (J2000.0: $\alpha=04 \mathrm{~h}: 09 \mathrm{~m}: 17 \mathrm{~s} .0, \delta=+30^{\circ}: 46^{\prime}: 33.5^{\prime \prime}$, Galactic
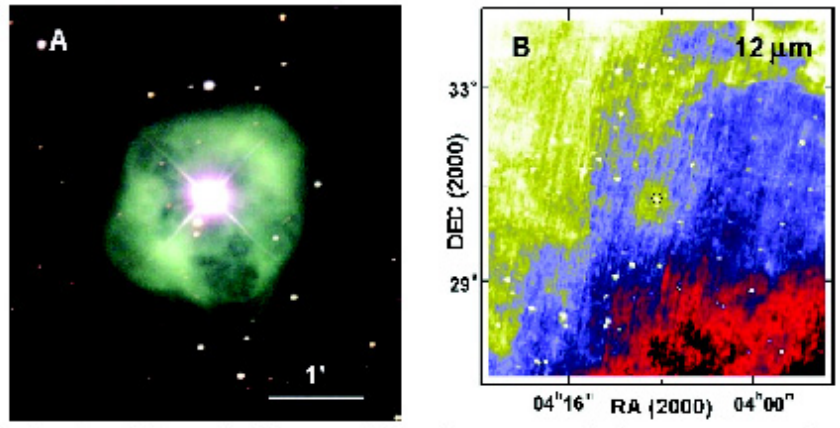

Fig. 1: (A) Optical image of the planetary nebula NGC 1514. The bright object at the nebula's center is the A0III star HD 281679, the companion star of the spectroscopically confirmed (13) hot $\left(\sim 6 \times 10^{4}\right.$ K) central star, which has not been detected hitherto on direct images. North is up, east to the left. Note the brightness depression in the nebula and the faint protrusion of nebular emission towards the southsouth-west, at a position angle $P A \approx 197^{\circ} \pm 5^{\circ}$. (B) The area surrounding NGC 1514 in the infrared. Shown is a $7.5^{\circ}$ region at 12 $\mu \mathrm{m}$, taken from IRAS using SkyView, centered on the PN (circle; being $\sim 5 \times$ larger than the PN). NGC 1514 is located close to the center of a large $\left(\sim 0.8^{\circ}\right)$ roundish dust emission region.

coordinates $l=165.53^{\circ}, b=-15.29^{\circ}$ ) is a bright roundish amorphous PN with a radius of $\sim 65^{\prime \prime}$ and has a faint halo with a radius of $\sim 90$ " (10). It harbors a binary: the bright companion to the true, invisible, central star is of type AOII and has a proper motion of $\mu_{\alpha} \cdot \cos \delta=-8.14 \pm 1.54$ and $\mu_{\delta}=3.63 \pm 1.24$ milliarcsec/yr (11). Since this star is (still) centrally located, one may safely assume that this proper motion also applies to the planetary nebula. In Fig. 1A an optical image of the nebula and its $\mathrm{AOII}$ star is reproduced.

Awthorfor Correspondeno: B. Aryal, Central Department of Physics, Tribhuvan University, Kirtipur, Kathmandı, Nepal. 
NGC 1514 is one of the few PNe with arelatively reliable distance of $D=185(+140,-30) \mathrm{pc}(11)$. In the framework of a spatiokinematic study it is argued that the nebula is a descendant of a common envelope system and that the binary period is 4 9 days, with an initial (i.e. main sequence) mass of the progenitor of the true (hot) central star of $\sim 4.5 M_{N}$ (12). Since central stars of PNe representyoung white dwarfs with a Chandrasekhar (upper)

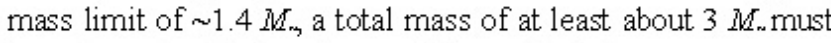
have been shed during the evolution of this star.

In Fig. $1 \mathrm{~B}$ an image in the IRAS $12 \mu \mathrm{m}$ bandpass of a large area around the PN shows NGC 1514 to be located near the middle of a roundish region of enhanced homogeneous emission with a diameter of $\sim 0.8^{\circ}$. NGC 1514 itself clearly stands out as a point-like emission peak (Fig. 1B). Both the shape of the region and the location of the PN suggest a relation between these two. We assume that the emission is due to dust and that the region is at $D=185 \mathrm{pc}$. By use of the $60 \mu \mathrm{m}$ and $100 \mu \mathrm{m}$ fluxes of this region (after subtraction of the infrared emission of NGC 1514) we found a color temperature of $\mathrm{T}=29 \pm 3 \mathrm{~K}$, using (14). From the $\mathbb{R}$ fluxes (in Jy) of the PN (12 $\mu \mathrm{m}:<0.25 ; 25 \mu \mathrm{m}: 1.03 \% ; 60 \mu \mathrm{m}: 10.17$; and 100 $\mu \mathrm{m}: 21.97$ ) the $60 \mu \mathrm{m}$ and $100 \mu \mathrm{m}$ fluxes lead to the same temperature, i.e. $T=29 \pm 3 \mathrm{~K}$, but there is also a much warmer component $(>100 \mathrm{~K}$ ) derived from the 12 and $25 \mu \mathrm{m}$ data (all these data have been derived by use of IRAS images from Groningen). A temperature of $29 \mathrm{~K}$ - only several degrees higher than the interstellar cirrus temperature of $\sim 20 \mathrm{~K}$-points to dust heating from the outside, probably from the interstellar radiation field plus a contribution from the inner nebula and its central source(s)

\section{RESULTS}

Assuming a gas-to-dust mass ratio of 150 and using a commonly accepted dust mass relation (15), we derived total masses of $2.1 \pm 1.4 M$. for the roundish region (without NGC 1514) and $0.08 \pm 0.05 M_{\text {. }}$ for the PN. The combined mass hence is about $2.2 \pm 1.4 \mathrm{M}$, comparable to the estimate of the total mass shed by the progenitor of NGC 1514 (12). On high resolution (HIRES) images of IRAS maps (HIRES can produce resolution of $\sim 1^{\prime}$ ) of the roundish region, symmetrical emission structures around NGC 1514 become visible. From now on we will as sume that all these structures are linked to the PN and lie in the plane of the sky. The latter as sumption will for example lead to lower limits on physical sizes. In Fig. 2 the brighter of these are presented in the four IRAS passbands. The least disturbed is the one at $100 \mu \mathrm{m}$, which is of symmetrical shape, reminding of the appearance of a bipolar outflow. In Fig. 2D this bipolar structure has a $100 \mu \mathrm{m}$ angular extent of 22 , corresponding to a linear size of $1.2 \mathrm{pc}$. Its length-to-width ratio is $\sim 3$. Adistinct peak of emission is present in the north, 8.1' $(0.44 \mathrm{pc})$ distant from NGC 1514

The long axis of the whole structure shows a position angle $P A=200^{\circ} \pm 2^{\circ}$, i. e. practically the same $P A$ (within the errors) as the brightness depression in the nebula and the faint

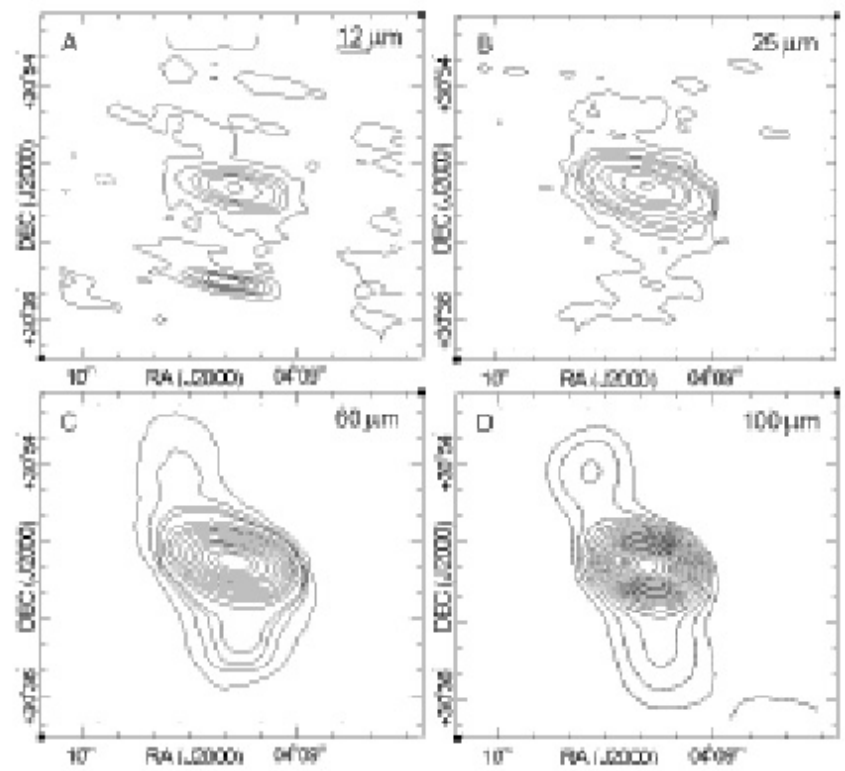

Fig. 2: Contour maps of a $\sim 0.5^{\circ} \times \sim 0.5^{\circ}$ region in the infrared centered on NGC 1514. The IRAS (HIRES) contours $\mathrm{span}$, in panel (A) 0.0 to $2.0 \mathrm{MJy} / \mathrm{sr}$ as follows: $0,0.27,0.57,0.87,1.17,1.47$, and 2.0; in panel (B) 0.5 to $8.0 \mathrm{MJy} / \mathrm{sr}$ as follows: $0.5,0.8,1.1,1.4,2$, $3.5,4.5,6.0$, and 8.0 ; in panel (C) 1.05 to $17.0 \mathrm{MJy} / \mathrm{sr}$ in the steps $1.05,1.3,1.5,1.65,2.25,2.85,3.5,4.5,6.0,8.0,10.0,12.0,15.0$, and 17.0 , and in panel (D) 6.7 to $17.1 \mathrm{MJy} / \mathrm{sr}$, in steps separated by $0.65 \mathrm{MJy} / \mathrm{sr}$, respectively. The slight east-west elongation of the contours is not real. Particularly at $12 \mu \mathrm{m}$ there is a prominent background source $7.5^{\prime}$ south of the center of the PN. This source twists the southern part of the bipolar lobe, but least (or not at all) at $100 \mu \mathrm{m}$. The arrow shows the direction of the proper motion of the A0III star.

protrusion of nebular emission towards the south-south-west in the PN itself (Fig. 1A). This long axis, defined as a straight line passing through the center of the northern peak of emission and dividing the southern lobe in two halves of about equal area lies east of the PN (offset 1.4', corresponding to $0.075 \mathrm{pc}$ ) and is perpendicular to the proper motion of the PN. The offset can, provided the structure represents an outflow, be due to an interaction with ambient medium. In Fig. 2D the contours of NGC 1514 show a steeper gradient towards the direction of proper motion, supporting an interaction. The mass of the structure - after subtraction of the PN and of the fainter matter within which it is embedded (see below) - was estimated to be $0.24 \pm 0.16 M_{n}$ (using $T=29$ K). No object (galaxy, dust-enshrouded star...) that could be the source of the peak of infrared emission in the northem lobe was found.

When going to fainter brightness levels on the HIRES 100 $\mu \mathrm{m}$ images, two additional outer emission peaks become visible (Fig. 3). They are located in the north-east and southwest, respectively, and have a separation from NCG 1514 of $\sim 20^{\prime}$ each. Astraight line connecting these peaks shows a $P A$ $=218^{\circ}$ and clearly lies east of the PN (offset $1.8^{\prime}$, corresponding to $0.097 \mathrm{pc}$ at $D=185 \mathrm{pc}$ ). Hence, this straight line lies slightly eastwards of the long axis of the inner structure described 
above, i. e. farther away from the PN compared to the inner structure. Again we believe that this offset is due to an interaction with ambient medium, supported by the gradient in the contours towards the direction of motion of the PN (Fig. 3). The peaks of this outer structure have a linear separation of $2.1 \mathrm{pc}$. The total mass of the outer structure could be estimated in a very approximate way only: we found $0.4 \pm 0.3 M_{\text {sun }}$ (using $T=29 \mathrm{~K}$ ), that is it appears to have roughly the same mass as the inner structure. No objects that could be sources of the peaks of infrared emission in the lobes were found. In the optical, there is very faint widespread diffuse emission visible around NGC 1514 on the Palomar Observatory Sky Survey I and II with a surface brightness fainter than $\sim 24 \mathrm{mag} / \operatorname{arcsec}^{2}$, possibly (in part) representing extensions of reflecting dust clouds from the star forming regions Tau I and II that are $\sim 3^{\circ}$ away in the south-east. An optical spectrum ( $45 \mathrm{~min}, 1.8 \mathrm{~m}$ telescope of the Asiago Observatory) of the northern lobe, compared to an analogous spectrum north of this lobe did not show any noticeable line emission.
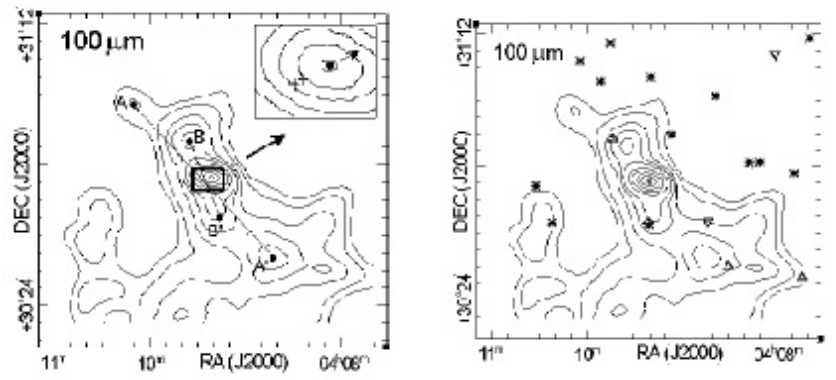

Fig. 3(A): Contour map of a $\sim 1^{\circ} \times \sim 1^{\circ}$ region at $100 \mu \mathrm{m}$ centere $d$ on NGC 1514. The IRAS (HIRES) contours represent 3.7, 4.5, 5.8, 6.8, $7.8,10.0,12.0,14.0,16.0$ and $17.5 \mathrm{MJy} / \mathrm{sr}$, respectively. $\mathrm{A}$ and $\mathrm{A}^{\prime}$ mark the maxima of the outer structure, $B$ and $B^{\prime}$ the maxima of the inner structure. They are connected by straight lines, thus demonstrating their offset from the position of NGC 1514. The insert shows a magnification of the inner region of the figure (red box), revealing that the midpoints (blue plus signs) of the lines connecting $\mathrm{A}-\mathrm{A}^{\prime}$ and $\mathrm{B}-\mathrm{B}^{\prime}$ lie along the direction of the proper motion (arrow) of NGC 1514, the position of which is marked by an asterisk. (B) Known discrete sources (from the SIMBAD data base) projected onto the $100 \mu \mathrm{m}$ IRAS (HIRES) contour map. The field is the same as that of $(A)$. The stars, triangles, upside down triangles, diamonds, and the plus sign represent stars, IRAS sources, X-ray sources, Ced 29 (nebula of unknown nature) and NGC 1514, respectively.

This star forming complex is, at $D=140 \mathrm{pc}$, closer to us than NGC 1514, and the PN might lie behind some outlying traces of molecular (and probably dusty) material of it ( 26 ). Hence, we cannot exclude that the $0.8^{\circ}$ large roundish nebula in Fig. $1 \mathrm{~B}$ is a foreground object or is slightly contaminated by foreground material. The shape of this nebula and the location of the PN however rather suggest a stellar origin. As is evident from Figs. 2 and 3, the well defined bipolar inner structure and the outer dust emission structure could be contaminated too, but the former almost certainly appears to be physically linked to the central object, i. e. the PN and/or its progenitor.

NGC 1514 has an average expansion velocity of $23 \mathrm{~km} / \mathrm{s}$ (12). Taking its radius of $65^{\prime \prime}$ (that is $0.058 \mathrm{pc}$ at $D=185 \mathrm{pc}$ ), its average expansion velocity of $23 \mathrm{~km} / \mathrm{s}$ (12), and assuming constant expansion velocity, the planetary nebula is not older than about 2,500 years.

\section{DISCUSSION}

We suggest that all the structures described above can be put in chronological order. The roundish nebula is the fossil remnant of the spherically symmetric outflow in the AGB phase. The outer bipolar structure marks the first (massive) aspherical ouflow in the PPN phase, later followed by a second one (the inner bipolar structure), which took place along another axis due to precession. Later, the PN formed and was partly shaped by the effects of the earlier dusty outflows as is indicated by its brightness depression in along the same axis as the inner bipolar structure. These collimated outflows are probably driven by an accretion disk around the hot compact central star or around a possible third companion star; NGC 1514 perhaps might contain a triple-star system (5). To sum up, NGC 1514 and its surroundings could represent the preserved history of the main mass loss phases of a star of interme diate initial mass.

Typical wind velocities in the AGB phase are $10-20 \mathrm{~km} / \mathrm{s}$. To cover the angular distance of $0.4^{\circ}$ (corresponding to $1.29 \mathrm{pc}$ at $D=185 \mathrm{pc}$ ) with a constant velocity of $15 \mathrm{~km} / \mathrm{s}$ takes $8.4 \times$ $10^{4}$ years. Hence this is approximately the time when the strong spherical AGB mass loss started; its material is now visible as the roundish nebula of dust emission. In the short (typically $\sim 1,000$ years) phase of transformation from spherical mass loss into an aspherical nebula, the outer structure ( $A$ - $A^{\prime}$ in Fig. 3) was generated; it may contain entrained material from the $\mathrm{AGB}$ wind. We assume that the generation of the outer structure started 1,000 years before the PN was born, i.e. 3,500 years ago. To cover $19.8^{\prime}(1.07 \mathrm{pc})$ within 3,500 years requires a velocity of $298 \mathrm{~km} / \mathrm{s}$ (being a lower limit in case that this structure is not in the plane of the sky). Speeds of a few $100 \mathrm{~km} / \mathrm{s}$ are indeed characteristic for collimated bipolar outflows in this phase $(4,17-19)$. We further assume that the inner outflow (Fig. 2D) started 3,000 years ago: To bridge the distance between the midpoints of the line $\mathrm{B}-\mathrm{B}^{\prime}$ (Fig. 3A) to the northern peak ( $8.1^{\prime}$, corresponding to $0.44 \mathrm{pc}$ ) would result in a velocity of (at least) $143 \mathrm{~km} / \mathrm{s}$. The offsets of the midpoints of the lines $A-A^{\prime}$ and $B-B^{\prime}$ along the proper motion vector of the PN (i.e. the ir lagging behind) could indicate interaction with ambient matter, but due to the long time scales involved any non-alignment with the position of the PN also cannot be ruled out. Finally, the channel-like reduction in brightness in the south-south-west in the optical image of NGC 1514 (Fig. 1A) might either be an indication of a still ongoing mild mass loss - a part of the ionized gas in the PN appears to use this channel to protrude from the main body of the PN. Alternatively, this cavity could stem from the shock interaction among different winds, resulting from the mass loss process already finished, i.e. where the most collimated component already escaped from the nebula.

\section{CONCLUSION}

We suspect that this planetary nebula is not the only one where fossil records of (bipolar) ancient mass loss in its 
neighbourhood as well as signs of the resulting shaping of the PN can be found. Due to its closeness, the rather smooth infrared background emission and particularly due to the high mass lost by its progenitor it however appears to offer optimum conditions for detection and further study.

\section{ACKNOWLEDGEMENTS}

This work was supported by the Austrian Science Fund (FWF), project no. P15316. We thank Drs. S. Kimeswenger and $\mathrm{A}$ Castro-Carrizo for the ir comments and suggestions. We are grateful to Dr. S. G. Temporin for her help in observing at the Cima Ekar Telescope of the Asiago Observatory. One of the authors (AM) acknowledges Central Department of Physics, Tribhuvan University, Kirtipur, for providing various forms of support for his masters thesis.

\section{REFERENCES}

1. Kwok, S. 1998. Sky \& Tel. 96: 30 .

2. Weinberger, R. and Kerber, F. 1997. Science 276:1382.

3. Balick, B. A. and Frank. 2002. Ann. Rev. Astron. Astrophys. 40:439.

4. Sahai, R. et al. 2003. Nature. 426: 261.

5. Soker, N. 2004. Mon. Not. R. Astron. Soc. 350: 1366.
6. Vinkovic, D. 2004. et al, Mon. Not. R. Astron. Soc. 352, 852 .

7. Henning Th. and Salama, F. 1998. Science 282: 2204.

8. Kwok, S., Su, K.Y. L and Sahai, R. 2000. Bull. Am. Astron. Soc. 32: 1397.

9. Sahai, R. 2002. Rev. Mex. Astron. Astrophys. 13:133.

10. Chu, Y.-H., Jacoby, GH. and Arendt, R. 1987. Astrophys. J. Suppl. Ser. $64: 529$

11. Acker, A., Fresneau, A., Pottasch, S.R. and Jasniewicz, G 1998. Astron. Astrophys. 337: 253.

12. Muthu, C. and Anandarao, B.G 2003. Astron. J. 126: 2963.

13. Seaton, M. 1980. Quart. J. R Astron. Soc. 21: 229.

14. Henning, Th., Pfau, W. and Altenhoff, W.J. 1990. Astron. Astrophys. 227: 542 .

15. Hildebrand, R.H. 1983. Quart. J. R Astron. Soc. 24: 267.

16. Briceno, C., Hartmann, L., Stauffer, J. and Martin, E. 1998. Astron. J. 115, 2074.

17. Imai, H. et al. 2002. Nature 417: 829.

18. Castro-Carrizo, A. et al. 2002. Astron. Astrophys. 386: 633

19. Alcolea J. et al. 2001. Astron. Astrophys. 373: 932. 